PROCEEDINGS OF THE

AMERICAN MATHEMATICAL SOCIETY

Volume 136, Number 8, August 2008, Pages 2647-2654

S 0002-9939(08)09289-7

Article electronically published on April 11, 2008

\title{
A NOTE ON RESOLUTION OF RATIONAL AND HYPERSURFACE SINGULARITIES
}

\author{
D. A. STEPANOV
}

(Communicated by Ted Chinburg)

\begin{abstract}
It is well known that the exceptional set in a resolution of a rational surface singularity is a tree of rational curves. We generalize the combinatoric part of this statement to higher dimensions and show that the highest cohomologies of the dual complex associated to a resolution of an isolated rational singularity vanish. We also prove that the dual complex associated to a resolution of an isolated hypersurface singularity is simply connected. As a consequence, we show that the dual complex associated to a resolution of a 3-dimensional Gorenstein terminal singularity has the homotopy type of a point.
\end{abstract}

\section{INTRODUCTION}

Let $o \in X$ be an isolated singularity of an algebraic variety (or an analytic space) $X$ defined over a field of characteristic $0, \operatorname{dim} X \geq 2$. Consider a good resolution $f: Y \rightarrow X$ (this means that the exceptional locus $Z \subset Y$ of $f$ is a divisor with simple normal crossings). Let $Z=\sum Z_{i}$, where $Z_{i}$ are irreducible. To the divisor $Z$ we can associate the dual complex $\Gamma(Z)$. It is a CW-complex whose cells are standard simplexes $\Delta_{i_{0} \ldots i_{k}}^{j}$ corresponding to the irreducible components $Z_{i_{0} \ldots i_{k}}^{j}$ of the intersections $Z_{i_{0}} \cap \cdots \cap Z_{i_{k}}=\bigcup_{j} Z_{i_{0} \ldots i_{k}}^{j}$. The $k-1$-simplex $\Delta_{i_{0} \ldots \hat{i_{s} \ldots i_{k}}}^{j^{\prime}}$ is a face of the $k$-simplex $\Delta_{i_{0} \ldots i_{k}}^{j}$ iff $Z_{i_{0} \ldots i_{k}}^{j} \cap Z_{i_{0} \ldots \hat{i_{s} \ldots i_{k}}}^{j^{\prime}} \neq \varnothing$. If $X$ and $Y$ are surfaces, then $\Gamma(Z)$ is the usual resolution graph of $f$. Note that $\Gamma(Z)$ is a simplicial complex iff all the intersections $Z_{i_{0}} \cap \cdots \cap Z_{i_{k}}$ are irreducible. This can be obtained for a suitable resolution. Also note that if $\operatorname{dim} X=n$, then $\operatorname{dim}(\Gamma(Z)) \leq n-1$.

The complex $\Gamma(Z)$ was first studied by G. L. Gordon in connection to the monodromy in families (see [7). We say that $\Gamma(Z)$ is the dual complex associated to the resolution $f$. The main reason motivating the study of the dual complex is the fact that the homotopy type of $\Gamma(Z)$ depends only on the singularity $o \in X$

Received by the editors March 20, 2006 and, in revised form, July 2, 2006 and November 16, 2006.

2000 Mathematics Subject Classification. Primary 14B05; Secondary 32S50.

Key words and phrases. Rational singularity, hypersurface singularity, resolution of singularities, the dual complex associated to a resolution.

This research was supported by RFBR, grant no. 05-01-00353, CRDF, grant no. RUM12692-MO-05, and the Program for the Development of Scientific Potential of the High School, no. 2.1.1.2381.

(C)2008 American Mathematical Society Reverts to public domain 28 years from publication 
but not on the choice of a resolution $f$. This is a consequence of the AbramovichKaru-Matsuki-Włodarczyk Weak Factorization Theorem in the Logarithmic Category (see [1]). Indeed, this theorem reduces the problem to the case of a blowup $\sigma:\left(X^{\prime}, Z^{\prime}\right) \rightarrow(X, Z)$, where $X$ and $X^{\prime}$ are smooth varieties with divisors $Z$ and $Z^{\prime}$ with simple normal crossings, and the center of the blowup is admissible in some sense. It can be explicitly verified that $\Gamma(Z)$ is homotopy equivalent to $\Gamma\left(Z^{\prime}\right)$ (see [16]).

For example (it is taken from [7]), consider the singularity

$$
\left\{x_{1}^{n+1}+x_{2}^{n+1}+\cdots+x_{n}^{n+1}+x_{1} x_{2} \ldots x_{n}=0\right\} \subset \mathbb{C}^{n} .
$$

A good resolution can be obtained just by blowing up the origin. The reader can easily prove that the exceptional divisor $Z$ consists of $n$ hyperplanes in a general position in $\mathbb{P}^{n-1}$. We see that the complex $\Gamma(Z)$ is the border of a standard $n-1$ dimensional simplex, and thus it has the homotopy type of the sphere $S^{n-2}$.

If $F:(Y, Z) \rightarrow(X, o)$ is a resolution of an isolated toric singularity $(X, o)$, then the complex $\Gamma(Z)$ has the homotopy type of a point ([16]).

In this paper, we study the dual complex associated to a resolution in the case when $X$ is a rational or a hypersurface singularity defined over the field $\mathbb{C}$ of complex numbers. We show that if $f: Y \rightarrow X$ is a good resolution of an isolated rational singularity $o \in X, \operatorname{dim} X=n$, then $H^{n-1}(\Gamma(Z), \mathbb{C})=0$ (see Theorem 2.2). The proof is a generalization of M. Artin's argument from [3] to the $n$-dimensional case. The main new ingredient is the lemma on the degeneracy of a spectral sequence associated to a divisor with simple normal crossings on a Kähler manifold (Lemma 2.4). If $X$ is an isolated hypersurface singularity, $\operatorname{dim} X \geq 3$, then $\pi(\Gamma(Z))=0$ (see Theorem 3.1). This result is based on the well known fact that the link of an isolated hypersurface singularity of dimension $n \geq 3$ is simply connected (14]). These results allow us to prove that the homotopy type of the dual complex associated to a resolution of an isolated rational hypersurface 3-dimensional singularity is trivial (Corollary 3.3). As an application, we show that the dual complex associated to a resolution of a 3-dimensional Gorenstein terminal singularity has the homotopy type of a point (Corollary 3.4).

We prove our theorems for algebraic varieties, but everything also holds for analytic spaces (with obvious changes).

The author is grateful to V. A. Iskovskikh, K. Matsuki, Yu. G. Prokhorov, J. Steenbrink and J. Whal for written and oral consultations that were very stimulating and useful. We also thank P. Popescu-Pampu who noticed a mistake in the first version of the paper and to the referee who suggested several improvements to the text.

\section{Rational Singularities}

Recall

Definition 2.1. An algebraic variety (or an analytic space) $X$ has rational singularities if $X$ is normal and for any resolution $f: Y \rightarrow X$ all the sheaves $R^{i} f_{*} \mathcal{O}_{Y}$ vanish, $i>0$.

In the sequel, when we say that $f$ is a good resolution we additionally assume that all the intersections $Z_{i_{0}} \cap \cdots \cap Z_{i_{k}}$ of prime components of the exceptional divisor $Z=\sum Z_{i}$ of $f$ are irreducible, thus $\Gamma(Z)$ is a simplicial complex. 
The following theorem can be considered as a generalization of the classical fact that the exceptional locus in a resolution of a rational surface singularity is a tree of rational curves ([3]).

Theorem 2.2. Let $o \in X$ be an isolated rational singularity of a variety (or an analytic space) $X$ of dimension $n \geq 2$, and let $f: Y \rightarrow X$ be a good resolution with the exceptional divisor $Z$. Then the highest (complex) cohomologies of the complex $\Gamma(Z)$ vanish:

$$
H^{n-1}(\Gamma(Z), \mathbb{C})=0 .
$$

Proof. Let $Z=\sum_{i=1}^{N} Z_{i}$ be the decomposition of the divisor $Z$ to its prime components $Z_{i}$. We can assume that $X$ is projective (since the given singularity is isolated) and $f$ is obtained by a sequence of smooth blowups (Hironaka's resolution 12]). Thus all $Z_{i}$ and $Y$ are Kähler manifolds.

The sheaves $R^{i} f_{*} \mathcal{O}_{Y}$ are concentrated at the point $o$. Via Grothendieck's theorem on formal functions (see [11], (4.2.1), and [9], Ch. 4, Theorem 4.5 for the analytic case) the completion of the stalk of the sheaf $R^{i} f_{*} \mathcal{O}_{Y}$ at the point $o$ is

$$
\lim _{(r) \rightarrow(\infty)} H^{i}\left(Z, \mathcal{O}_{Z_{(r)}}\right)
$$

where $(r)=\left(r_{1}, \ldots, r_{N}\right)$ and $Z_{(r)}=\sum_{i=1}^{N} r_{i} Z_{i}$. If $(r) \geq(s)$, i. e., $r_{i} \geq s_{i} \forall i$, there is a natural surjective map $g$ of sheaves on $Z$ :

$$
g: \mathcal{O}_{Z_{(r)}} \rightarrow \mathcal{O}_{Z_{(s)}}
$$

Since dimension of $Z$ is $n-1$, the map $g$ induces a surjective map of cohomologies

$$
H^{n-1}\left(Z, \mathcal{O}_{Z_{(r)}}\right) \rightarrow H^{n-1}\left(Z, \mathcal{O}_{Z_{(s)}}\right) .
$$

Recall that the given singularity $o \in X$ is rational, and thus the projective limit (11) is 0 . Therefore the cohomology group $H^{n-1}\left(Z, \mathcal{O}_{Z}\right)$ vanishes, too (because the projective system in (1) is surjective). Now it follows from Lemma 2.3 below that $H^{n-1}(\Gamma(Z), \mathbb{C})=0$.

Lemma 2.3. Let $Z=\sum Z_{i}$ be a reduced divisor with simple normal crossings on a compact Kähler manifold $Y$, $\operatorname{dim} Y=n$, and assume that $H^{k}\left(Z, \mathcal{O}_{Z}\right)=0$ for some $k, 1 \leq k \leq n-1$. Then the $k$-th cohomologies with coefficients in $\mathbb{C}$ of the complex $\Gamma(Z)$ vanish, too:

$$
H^{k}(\Gamma(Z), \mathbb{C})=0 \text {. }
$$

Proof. Let us introduce some notation. Let $Z^{0}=\bigsqcup_{i} Z_{i}$ be the disjoint union of the irreducible components $Z_{i}$ and $Z^{p}=\bigsqcup_{i_{0}<i_{1}<\cdots<i_{p}} Z_{i_{0} i_{1} \ldots i_{p}}$ where $Z_{i_{0} i_{1} \ldots i_{p}}=$ $Z_{i_{0}} \cap Z_{i_{1}} \cdots \cap Z_{i_{p}}$. By $\varphi_{p}: Z^{p} \rightarrow Z$ denote the natural map. Consider the structure sheaves $\mathcal{O}_{Z^{p}}=\bigoplus_{i_{0}<\cdots<i_{p}} \mathcal{O}_{Z_{i_{0} \ldots i_{p}}}$, the sheaves $\mathcal{A}^{p, q}=\bigoplus_{i_{0}<\cdots<i_{p}} \mathcal{A}_{Z_{i_{0} \ldots i_{p}}}^{p, q}$ of differential forms of bidegree $(0, q)$ on $Z^{p}$ and their direct images $\mathcal{K}^{p}=\varphi_{p *} \mathcal{O}_{Z^{p}}$ and $\mathcal{K}^{p, q}=\varphi_{p *} \mathcal{A}^{p, q}$ on $Z$. The sequence of sheaves $\left\{\mathcal{K}^{p}\right\}_{p \geq 1}$ forms a complex via the combinatoric differentials $\delta^{p}: \mathcal{K}^{p} \rightarrow \mathcal{K}^{p+1}$, where if

$$
a=\bigoplus a_{i_{0} \ldots i_{p}} \in \mathcal{K}^{p}(U)=\bigoplus_{i_{0}<\cdots<i_{p}} \varphi_{p *} \mathcal{O}_{Z_{i_{0} \ldots i_{p}}}(U)
$$

is a section of the sheaf $\mathcal{K}^{p}$ over an open set $U \subseteq Z$, then

$$
(\delta(a))_{i_{0} \ldots i_{p} i_{p+1}}(U)=\left.\sum_{j=0}^{p+1}(-1)^{j}\left(a_{i_{0} \ldots \widehat{i_{j} \ldots i_{p+1}}}\right)\right|_{Z_{i_{0} \ldots i_{p+1}} \cap U} .
$$


Note that there is also a natural injection of $\mathcal{O}_{Z}$ into $\mathcal{K}^{0}$. The sequence of sheaves

$$
0 \rightarrow \mathcal{O}_{Z} \rightarrow \mathcal{K}^{0} \stackrel{\delta^{0}}{\rightarrow} \mathcal{K}^{1} \stackrel{\delta^{1}}{\rightarrow} \ldots
$$

is exact. This is easy to check by considering the stalks; in particular, the exactness at $\mathcal{K}^{0}$ is a consequence of the following fact which holds locally in a sufficiently small neighborhood of every point of $Z$ : if $\left\{f_{i}\right\}$ is a collection of regular functions on $Z_{i}$ such that their restrictions onto intersections $Z_{i} \cap Z_{j}$ coincide, then there exists a regular function $f$ on $Z$ such that $\left.f\right|_{Z_{i}}=f_{i}$ for all $i$ (it is important here that the divisor $Z$ has normal crossings). Therefore the complexes

$$
\mathcal{O}^{*}: \quad \mathcal{O}_{Z} \rightarrow 0 \rightarrow 0 \rightarrow \ldots
$$

and

$$
\mathcal{K}^{*}: \quad \mathcal{K}^{0} \stackrel{\delta^{0}}{\rightarrow} \mathcal{K}^{1} \stackrel{\delta^{1}}{\rightarrow} \mathcal{K}^{2} \stackrel{\delta^{2}}{\rightarrow} \ldots
$$

are quasi-isomorphic. It is clear that the hypercohomologies of the first complex are isomorphic to the cohomologies of $Z$ with coefficients in the structure sheaf: $\mathbf{H}^{p}\left(\mathcal{O}^{*}\right) \simeq H^{p}\left(Z, \mathcal{O}_{Z}\right)$. Now let us calculate the hypercohomologies of the complex $\mathcal{K}^{*}$ by using the acyclic resolutions

$$
\mathcal{K}^{p} \rightarrow \mathcal{K}^{p, 0} \stackrel{\bar{\partial}}{\rightarrow} \mathcal{K}^{p, 1} \stackrel{\bar{\partial}}{\rightarrow} \ldots,
$$

where $\bar{\partial}$ is the Dolbeaux differential.

Consider the bigraded sequence of groups

$$
K^{p, q}=H^{0}\left(\mathcal{K}^{p, q}, Z\right) \simeq \bigoplus_{i_{0}<\cdots<i_{p}} H^{0}\left(\varphi_{p *} \mathcal{A}^{p, q}\right)
$$

endowed with the differentials $\bar{\partial}: K^{p, q} \rightarrow K^{p, q+1}$ and $\delta: K^{p, q} \rightarrow K^{p+1, q}$, where $\bar{\partial}$ is the Dolbeaux differential and $\delta$ is the combinatoric differential defined as follows: if $\alpha=\bigoplus \alpha_{i_{0} \ldots i_{p}} \in K^{p, q}$, then

$$
(\delta(\alpha))_{i_{0} \ldots i_{p} i_{p+1}}=\left.\sum_{j=0}^{p+1}(-1)^{q+j}\left(\alpha_{i_{0} \ldots \widehat{i_{j} \ldots i_{p+1}}}\right)\right|_{Z_{i_{0} \ldots i_{p+1}}} .
$$

These differentials satisfy the equality $\bar{\partial} \delta+\delta \bar{\partial}=0$, thus $\left(K^{*, *}, \delta, \bar{\partial}\right)$ is a bicomplex. Let $\left(K^{*}, d\right), K^{n}=\bigoplus_{p+q=n} K^{p, q}, d=\delta+\bar{\partial}$ be the associated complex. Now we can write that $\mathbf{H}^{p}\left(\mathcal{K}^{*}\right)=H^{p}\left(\mathcal{K}^{*}, d\right)$.

There is a filtration

$$
F^{p} K^{n}=\bigoplus_{\substack{p^{\prime}+q=n \\ p^{\prime} \geq p}} K^{p^{\prime}, q}
$$

on the complex $K^{*}$. It is known (see [10, Ch. 3, section 5) that the spectral sequence $E_{r}$ associated to the filtration $F^{p} K^{n}$ converges to the cohomologies $H^{*}\left(K^{*}, d\right)$ and

$$
\begin{aligned}
& E_{0}^{p, q} \simeq K^{p, q} \\
& E_{1}^{p, q} \simeq H_{\bar{\partial}}^{q}\left(K^{p, *}\right) ; \\
& E_{2}^{p, q} \simeq H_{\delta}^{p}\left(H_{\bar{\partial}}^{q}\left(K^{*, *}\right)\right) .
\end{aligned}
$$


In particular,

$$
\begin{aligned}
E_{1}^{p, 0} & \simeq H_{\bar{\partial}}^{0}\left(\bigoplus_{i_{0}<\cdots<i_{p}} H^{0}\left(\varphi_{p *} \mathcal{A}^{p, *}\right)\right) \\
& \simeq \bigoplus_{i_{0}<\cdots<i_{p}} \mathbb{C} .
\end{aligned}
$$

Therefore the cochain complex

$$
0 \rightarrow E_{1}^{0,0} \stackrel{\delta}{\rightarrow} E_{1}^{1,0} \stackrel{\delta}{\rightarrow} \ldots
$$

is isomorphic to the cochain complex that one uses to calculate cohomologies of $\Gamma(Z)$ (here we denote by the same letter $\delta$ the map between cohomologies induced by the combinatoric differential). It follows that

$$
E_{2}^{p, 0} \simeq H^{p}(\Gamma(Z), \mathbb{C}) .
$$

We shall show that the spectral sequence $E_{r}$ degenerates in $E_{2}$. The method of the proof is based on the standard technique of the theory of the mixed Hodge structures. We learned this from [13], Chapter 4, §2. Also compare [8]. Since the result about $E_{r}$ can be of a particular interest, we state it as a separate lemma.

Lemma 2.4. Let $Z=\sum Z_{i}$ be a reduced divisor with simple normal crossings on a compact Kähler manifold $Y$, and let $E_{r}$ be the associated spectral sequence as described above. Then $d_{r}=0$ for all $r \geq 2$, i.e., this spectral sequence degenerates in $E_{2}$.

Proof. Consider the diagram

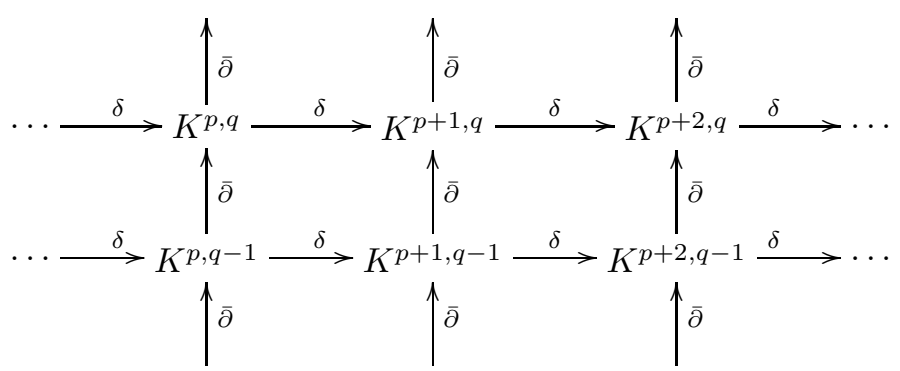

where $\bar{\partial} \delta+\delta \bar{\partial}=0$. First we take cohomologies in the vertical rows and obtain the sequences

$$
\begin{gathered}
\ldots \rightarrow H^{q}\left(K^{p, *}\right) \stackrel{\delta}{\rightarrow} H^{q}\left(K^{p+1, *}\right) \stackrel{\delta}{\rightarrow} H^{q}\left(K^{p+2, *}\right) \rightarrow \ldots \\
\cdots \rightarrow H^{q-1}\left(K^{p, *}\right) \stackrel{\delta}{\rightarrow} H^{q-1}\left(K^{p+1, *}\right) \stackrel{\delta}{\rightarrow} H^{q-1}\left(K^{p+2, *}\right) \rightarrow \ldots \\
\ldots \ldots
\end{gathered}
$$

Here $\delta$ is the induced map between cohomologies. Then we calculate $\delta$-cohomologies and obtain the differential

$$
d_{2}: H_{\delta}^{p}\left(H_{\bar{\partial}}^{q}\left(K^{*, *}\right)\right) \rightarrow H_{\delta}^{p+2}\left(H_{\bar{\partial}}^{q-1}\left(K^{*, *}\right)\right)
$$

that acts as described below. 
An element

$$
\overline{\bar{a}} \in H_{\delta}^{p}\left(H_{\bar{\partial}}^{q}\left(K^{*, *}\right)\right)
$$

is a class of those $\bar{a} \in H_{\bar{\partial}}^{q}\left(K^{p, *}\right)$ modulo $\delta\left(H_{\bar{\partial}}^{q}\left(K^{p-1, *}\right)\right)$ that map to 0 under the action of $\delta: \delta(\bar{a})=0$ in $H_{\bar{\partial}}^{q}\left(K^{p+1, *}\right)$. But $\bar{a}$ is a class of $a \in K^{p, q} \bmod \bar{\partial} K^{p, q-1}$ such that $\bar{\partial} a=0 \in K^{p, q+1}$. Therefore we can choose a representative $a \in K^{p, q}$ for $\overline{\bar{a}}$ such that $\bar{\partial} a=0$ and $\delta a=0$ modulo $\bar{\partial} K^{p+1, q-1}$ in $K^{p+1, q}$. It follows that there exists an element $a^{\prime} \in K^{p+1, q-1}$ such that $\bar{\partial} a^{\prime}=\delta a$. Map this $a^{\prime}$ down to $K^{p+1, q-1}: \delta\left(a^{\prime}\right) \in K^{p+2, q-1}$. It can be verified by standard methods that $\delta\left(a^{\prime}\right)$ correctly determines a class $\overline{\overline{\delta\left(a^{\prime}\right)}}$ in $H_{\delta}^{p+2}\left(H_{\bar{\partial}}^{q-1}\left(K^{*, *}\right)\right)$ and the differential $d_{2}$ is defined as follows:

$$
d_{2}(\overline{\bar{a}})=\overline{\overline{\delta\left(a^{\prime}\right)}} .
$$

Differentials $d_{r}, r \geq 3$, can be obtained by iterating this construction. For example, $d_{3}$ is defined for $a \in K^{p, q}$ such that $\delta\left(a^{\prime}\right)=0$ modulo $\bar{\partial} K^{p+2, q-1}$, thus there is an $a^{\prime \prime} \in K^{p+2, q-2}$ such that $\bar{\partial}\left(a^{\prime \prime}\right)=\delta\left(a^{\prime}\right)$, and $d_{3}: E_{3}^{p, q} \rightarrow E_{3}^{p+3, q-2}$ is induced by correspondence $a \rightarrow \delta\left(a^{\prime \prime}\right)$ (see, e.g., [5], A.3.13.4).

Our aim is to show that $d_{r}=0, r \geq 2$. The differential $d_{2}$ is trivial if the representative $a \in K^{p, q}$ can be chosen in such a way that $\delta(a)$ is exactly 0 but not only 0 modulo $\bar{\partial} K^{p+1, q-1}$. But this is true because there are harmonic differential forms in the class $\bar{a} \in H_{\bar{\partial}}^{q}\left(K^{p, *}\right)$ and we can take $a$ to be a harmonic form. The form $\delta a$ is defined by means of restrictions onto subvarieties and linear operations. All varieties we consider are Kähler, hence $\delta a$ is also harmonic. But it is $0 \bmod \left(\bar{\partial} K^{p+1, q-1}\right)$ and thus is exactly 0 . Further, this $\delta a=0$ can be lifted to $a^{\prime}=0$ in $K^{p+1, q-1}$, thus $\delta\left(a^{\prime}\right)=0$ and so forth. This also shows that $d_{r}=0$ for all $r \geq 3$.

Now let us come back to the proof of Lemma 2.3. We have $E_{2}=E_{\infty}$; therefore $H^{p}(\Gamma(Z), \mathbb{C}) \simeq E_{2}^{p, 0}$ is a subgroup of

$$
H^{p}\left(K^{*}, d\right) \simeq \mathbf{H}^{p}\left(K^{*}\right) \simeq \mathbf{H}^{p}\left(\mathcal{O}^{*}\right) \simeq H^{p}\left(Z, \mathcal{O}_{Z}\right) .
$$

If $H^{p}(\Gamma(Z))$ is not trivial, then $H^{p}\left(Z, \mathcal{O}_{Z}\right)$ is also not trivial.

\section{Hypersurface Singularities}

Theorem 3.1. Let $o \in X$ be an isolated hypersurface singularity of an algebraic variety (or an analytic space) $X$ of dimension at least 3 defined over the field $\mathbb{C}$ of complex numbers. If $f: Y \rightarrow X$ is a good resolution of $o \in X$ and $Z$ its exceptional divisor, then the fundamental group of $\Gamma(Z)$ is trivial:

$$
\pi(\Gamma(Z))=0 .
$$

Proof. Let $n$ be the dimension of $X$. We can assume that $X$ is a hypersurface in $\mathbb{C}^{n+1}$ and the singular point $o$ coincides with the origin. Consider the link $M$ of singularity $o \in X$, i.e., the intersection of $X$ with a sphere $S^{2 n+1}$ of sufficiently small radius around the origin. The link $M$ is an $(n-2)$-connected smooth manifold (14, Corollary 2.9, Theorem 5.2); in particular, $M$ is simply connected.

We can also consider $M$ as the border of a tubular neighborhood of the exceptional divisor $Z \subset Y$. It is known (see 2]) that there is a surjective map $\varphi: M \rightarrow Z$ whose fibers are tori. It follows that the induced map $\varphi^{*}: \pi(M) \rightarrow \pi(Z)$ is also surjective and hence $\pi(Z)=0$. 
It remains to show that $\pi(Z)=0$ implies $\pi(\Gamma(Z))=0$. It is enough to construct a surjective map $\psi: Z \rightarrow \Gamma(Z)$ with connected fibers. The following lemma is, essentially, a partial case of the general construction of a map from a topological space $Z=\bigcup Z_{i}$ to the nerve $\Gamma(Z)$ corresponding to the covering $\left\{Z_{i}\right\}$ (see [4], p. 355). As in section 2 we assume that the intersections $Z_{i_{0} \ldots i_{p}}$ are irreducible so that $\Gamma(Z)$ is a simplicial complex.

Lemma 3.2. Let $Z$ be a divisor with simple normal crossings on an algebraic veriety or an analytic space $X$, and let $\Gamma(Z)$ be the corresponding dual complex. Then there exists a map $\psi: Z \rightarrow \Gamma(Z)$ which is $(i)$ simplicial in some triangulations of $Z$ and $\Gamma(Z)$, (ii) surjective, and (iii) has connected fibers.

Proof. First, let us take a triangulation $\Sigma^{\prime}$ of $Z$ such that all the intersections $Z_{i_{0} \ldots i_{p}}$ are subcomplexes. Next we make the barycentric subdivision $\Sigma$ of $\Sigma^{\prime}$ and the barycentric subdivision of the complex $\Gamma(Z)$. Now let $v$ be a vertex of $\Sigma$ belonging to the subcomplex $Z_{i_{0} \ldots i_{p}}$ but not to any smaller subcomplex $Z_{i_{0} \ldots i_{p} i_{p+1}}$ :

$$
v \in Z_{i_{0} \ldots i_{p}}, \quad v \notin Z_{i_{0} \ldots i_{p} i_{p+1}} \forall i_{p+1} .
$$

Then let

$$
\psi(v)=\text { the center of the simplex } \Delta_{i_{0} \ldots i_{p}} .
$$

This determines the map $\psi$ completely as a simplicial map (depending on the triangulation $\Sigma^{\prime}$ ). It is clear from our construction that $\psi$ is surjective.

We claim that the map $\psi$ has connected fibers. Indeed, first observe that $\psi$ can be represented as a composition of topological contractions of connected subcomplexes

$$
\begin{gathered}
Z_{i_{0} \ldots i_{p}} \backslash \text { (the open neighborhoods of intersections } \\
\text { of } \left.Z_{i_{0} \ldots i_{p}} \text { with } Z_{i_{p+1}} \forall i_{p+1} \neq i_{0}, \ldots, i_{p}\right) .
\end{gathered}
$$

By an open neighborhood of a subcomplex $K$ we mean the union of interior points of all simplicial stars of $\Sigma$ with centers on $K$. All complexes in (2) are connected because the codimension of $Z_{i_{0} \ldots i_{p}} \cap Z_{i_{p+1}}$ in $Z_{i_{0} \ldots i_{p}}$ is 2 .

Further, the contraction of a subcomplex $K$ from (2) can be factored into oneby-one contraction of maximal simplexes of $K$. The preimage of every connected set under such a contraction is connected since the preimage of every simplex is a simplex. Therefore the map $\psi$ has all the needed properties.

Some important types of singularities are rational hypersurface. Combining Theorems 2.2 and 3.1 , we can obtain some precise results in the 3 -dimensional case.

Corollary 3.3. Let $o \in X$ be an isolated rational hypersurface singularity of dimension 3. If $f: Y \rightarrow X$ is a good resolution with the exceptional divisor $Z$, then the dual complex $\Gamma(Z)$ associated to the resolution $f$ has the homotopy type of a point.

Proof. We know from Theorems 2.2 and 3.1 that $\Gamma(Z)$ is simply connected and $H^{2}(\Gamma(Z), \mathbb{C})=0$. Since $\operatorname{dim} X=3$, we have $\operatorname{dim}(\Gamma(Z)) \leq 2$. Thus $H_{2}(\Gamma(Z), \mathbb{Z})=$ 0 . Now Corollary 3.3 follows from the Inverse Hurevich and Whitehead Theorems. 
For instance, 3-dimensional Gorenstein terminal singularities are exactly isolated compound $\mathrm{Du}$ Val points (up to an analytic equivalence; for details see [15]). Here it is sufficient to us that compound Du Val points are hypersurface singularities. On the other hand, terminal singularities (and, moreover, canonical) are rational (see [6]). Combining these results with Corollary 3.3, we get

Corollary 3.4. Let $o \in X$ be a 3-dimensional Gorenstein terminal singularity and let $f: Y \rightarrow X$ be a good resolution with the exceptional divisor $Z$. Then the dual complex $\Gamma(Z)$ of $f$ has the homotopy type of a point.

\section{REFERENCES}

[1] Abramovich, D., Karu, K., Matsuki, K., Włodarczyk, J. Torification and factorization of birational maps, J. Amer. Math. Soc. 15 (2002), 531-572. MR.1896232 (2003c:14016)

[2] A'Campo, N. La fonction zeta d'une monodromie, Comment. Math. Helvetici 50 (1975), 233-248. MR0371889 (51:8106)

[3] Artin, M. On isolated rational singularities of surfaces, Amer. J. Math. 88 (1966), 129-136. MR0199191 (33:7340)

[4] Dold, A. Lectures on Algebraic Topology, Springer-Verlag, 1972. MR0415602 (54:3685)

[5] Eisenbud, D. Commutative Algebra with a View toward Algebraic Geometry, Springer-Verlag, 1995. MR1322960 (97a:13001)

[6] Elkik, R. Rationalité des singularités canoniques, Invent. Math. 64 (1981), 1-6. MR621766 (83a:14003)

[7] Gordon, G. L. On a simplicial complex associated to the monodromy, Transactions of the AMS 261 (1980), 93-101. MR576865 (81j:32017)

[8] Gordon, G. L. On the degeneracy of a spectral sequence associated to normal crossings, Pacific J. Math. 90(2) (1980), 389-396. MR600638 (83f:32011)

[9] Grauert, H., et al. Several complex variables VII. Sheaf-theoretical methods in complex analysis, Encyclopedia of Mathematical Sciences, 74, Springer-Verlag, Berlin, 1994. MR1326617 (96k:32001)

[10] Griffiths, P., Harris, J. Principles of Algebraic Geometry, v. 1, John Wiley and Sons, New York, 1978. MR507725 (80b:14001)

[11] Grothendieck, A. Éléments de géométrie algébrique, Ch. III, Institut des Hautes Études Scientifiques, Publications Mathématiques, No. 11 (1961). MR0217085 (36:177c)

[12] Hironaka, H. Resolution of singularities of an algebraic variety over a field of characteristic zero. I, II, Annals of Math. 79 (1964), 109-326. MR0199184 (33:7333)

[13] Kulikov, Vik. S., Kurchanov, P. F. Complex algebraic varieties: periods of integrals and Hodge structures, Algebraic Geometry, III, 1-217. Encyclopaedia Math. Sci., 36, Springer, Berlin, 1998. MR 1602375

[14] Milnor, J. Singular Points of Complex Hypersurfaces, Annals of Mathematics Studies, 61, Princeton University Press, Princeton, NJ, 1968. MR0239612 (39:969)

[15] Reid, M. Minimal Models of Canonical 3-folds, Algebraic Varieties and Analytic Varieties, Adv. Studies in Pure Math., Kinokuniya and North-Holland (1983), V. 1, 131-180. MR715649 (86a:14010)

[16] Stepanov, D. A. A note on the dual complex associated to a resolution of singularities (Russian), Uspekhi Matem. Nauk 61(1) (2006), 185-186. MR2239783 (2007c:14010)

Department of Mathematical Modeling, Bauman Moscow State Technical UniverSity, Moscow 105005, Russia

E-mail address: dstepanov@bmstu.ru 